\title{
Early postnatal changes in the perfusion index in term newborns with subclinical chorioamnionitis
}

\author{
C De Felice, A Del Vecchio, M Criscuolo, A Lozupone, S Parrini, G Latini
}

Arch Dis Child Fetal Neonatal Ed 2005;90:F411-F414. doi: 10.1136/adc.2004.068882

See end of article for authors' affiliations

Correspondence to: Dr Latini, Division of Neonatology, Perrino Hospital, Clinical Physiology Institute (IFCCNR), National Research Council of Italy, Lecce Section, s.s. 7 per Mesagne, 72100 Brindisi, Italy; gilatini@tin.it

Accepted 17 February 2005 Published Online First 29 April 2005

\begin{abstract}
Background: Chorioamnionitis (HCA) in term newborns is often subclinical and associated with neonatal morbidity and mortality.

Objective: To assess the value of the pulse oximetry perfusion index (PI) in the early prediction of subclinical HCA in term newborns.

Methods: PI cut-off values were first identified in 51 term newborns with HCA and 115 matched controls, retrospectively categorised on the basis of placental histology (study phase 1). The PI thresholds obtained were subsequently tested on an unselected case series of 329 prospectively recruited, term newborns (study phase 2). PI was evaluated during the first five minutes after delivery. Initial illness severity and short term clinical outcomes were determined.

Results: In study phase 1, newborns with HCA had lower PI one and five minutes $(p<0.0001)$ after delivery, lower one minute Apgar score ( $p=0.017)$, lower cord blood base excess $(p=0.0001)$, together with higher rates of admission to neonatal intensive care unit $(p=0.0001)$ and endotracheal intubation ( $p=0.017)$, and higher SNAP-PE $(p<0.0001)$ and NTISS $(p<0.0001)$ scores than those without HCA. In the prospective validation phase of the study, the PI cut-off values generated (one minute $\leqslant 1.74$, five minutes $\leqslant 2.18$ ) showed $100 \%$ sensitivity, $99.4 \%$ specificity, $93.7 \%$ positive predictive value, and $100 \%$ negative predictive value in identifying subclinical HCA. Early identification of HCA was associated with a decreased rate of admission to intensive care $(p=0.012)$, as well as lower initial illness severity $(p \leqslant 0.0001)$ and therapeutic intensity $(p=0.0006)$ than the newborns with HCA in phase 1. Conclusion: These findings suggest that early PI monitoring is helpful in identifying HCA in term newborns.
\end{abstract}

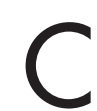
horioamnionitis is a typical disease of the second trimester of gestation and is associated with preterm birth with increased fetal and neonatal morbidity and mortality, as well as a higher risk of damage to the white matter of the brain and cerebral palsy. ${ }^{12}$ Little information on the effects of chorioamnionitis in the term fetus/newborn is available, although reported complications include depressed Apgar scores, neonatal encephalopathy with increased risk of developing cerebral palsy, ${ }^{2-6}$ infection, high risk of intubation in the delivery room, ${ }^{7}$ fetal death, ${ }^{8}$ and fetal growth restriction. ${ }^{9}$ A large proportion of cases of chorioamnionitis remain subclinical (histological chorioamnionitis (HCA) ), ${ }^{1}{ }^{10}{ }^{11}$ without sufficiently accurate clinical signs at birth being known to date. The pulsatile signal indexed against the non-pulsatile signal and expressed as a percentage $((\mathrm{AC} / \mathrm{DC}) \times 100)$ is commonly referred to as the perfusion index (PI). ${ }^{12}$ The pulse oximeter PI has been linked to increased illness severity and outcome in both newborns ${ }^{13}$ and adults. ${ }^{14}$ In this study, we postulated that changes in pulse oximeter PI early in life may occur in term newborns with subclinical HCA.

\section{METHODS}

To verify this hypothesis, PI cut-off values were first identified in a cohort of 51 term newborns (23 boys, 28 girls; mean (SD) gestational age 39.8 (1.3) weeks; birth weight 3128 (457) g) with HCA, and a matched control population of 115 newborns (54 boys, 61 girls; mean (SD) gestational age 39.5 (1.5) weeks; birth weight 3188 (572) g) without HCA, retrospectively categorised on the basis of placental histology, selected from an initial population of 500 consecutively born term infants (study phase 1). The PI cutoff values obtained were subsequently tested on an unselected case series of 329 consecutively born, term infants
(168 boys, 161 girls; gestational age 39.7 (1.2) weeks; birth weight 3320 (415) g), recruited prospectively (study phase 2).

PI was evaluated during the first five minutes after delivery, and initial illness severity and short term clinical outcomes were recorded. None of the mothers of the infants with HCA had clinical evidence of chorioamnionitis. The same methodology was applied to both phases ${ }^{12}$ of the study. Given the specific study aim of identifying the subclinical forms of placental inflammatory disease, three neonatologists were simultaneously involved during the study in the delivery room. The newborn caregiver was in charge of the infant's clinical conditions and primary resuscitation manoeuvres, and gave the Apgar scoring five and 10 minutes after birth. Independently, a second operator recorded the pulse oximetry signals during the first 10 minutes after birth, and the values obtained were stored in a laptop personal computer. PI was measured by applying the probe of the pulse oximeter to either foot of the infant, and artefact-free pulse wave signals were obtained 15.0 (2.5) seconds after birth. Median PI values, recorded at four second intervals during the time periods $0-1$ minute and 1-5 minutes, were used for statistical analysis. Finally, a third operator independently measured skin and core temperatures, clamped the umbilical cord, sent the placenta for histological examination, and performed the blood gas analyses. Initial illness severity was evaluated using the score for neonatal acute physiology-perinatal extension (SNAP-PE) and the neonatal therapeutic intervention scoring system (NTISS) 24 hours after admission. ${ }^{15} 16$

Abbreviations: $\mathrm{HCA}$, histological chorioamnionitis; $\mathrm{PI}$, perfusion index; NTISS, neonatal therapeutic intervention scoring system; SNAP-PE, score for neonatal acute physiology-perinatal extension 
Table 1 Results of screening for subclinical histological chorioamnionitis at one and five minutes by using perfusion index monitoring in two selected term newborn populations (phase 1 of the study)

\begin{tabular}{lll}
\hline & \multicolumn{2}{l}{ Histological chorioamnionitis } \\
\cline { 2 - 3 } Variables & Yes & No \\
\hline Number of cases & 51 & 115 \\
M:F & $23: 28$ & $54: 61$ \\
Gestational age (weeks) & $39.8(1.3)$ & $39.5(1.5)$ \\
Birth weight (g) & $3128(457)$ & $3188(572)$ \\
1 min perfusion index & $1.74(0.32)$ & $4.50(0.83)^{* * *}$ \\
5 min perfusion index & $2.18(1.02)$ & $4.42(2.10)^{* * *}$ \\
1 min Apgar score & $7(5-9)$ & $9(8-10)^{*}$ \\
5 min Apgar score & $9(8-10)$ & $9(9-10)$ \\
1 min core temperature ( $\left.{ }^{\circ} \mathrm{C}\right)$ & $37.02(0.15)$ & $37.05(0.05)$ \\
1 min skin temperature ( $\mathrm{C})$ & $34.50(0.22)$ & $34.54(0.25)$ \\
5 min core temperature ( $\left.{ }^{\circ} \mathrm{C}\right)$ & $37.01(0.25)$ & $37.03(0.31)$ \\
5 min skin temperature ( $\left.{ }^{\circ} \mathrm{C}\right)$ & $34.75(0.40)$ & $34.81(0.58)$ \\
Cord blood pH & $7.315(0.145)$ & $7.346(0.170)$ \\
Cord blood base excess & $-5.6(7.9)$ & $-1.7(4.1)^{* *}$ \\
Admission to NICU & $15(29.4 \%)$ & $7(6.1 \%)^{* *}$ \\
SGA (birth weight $<3 r d$ centile) & $5(9.8 \%)$ & $9(7.8 \%)$ \\
Endotracheal intubation & $5(9.8 \%)$ & $1(0.9 \%)^{*}$ \\
SNAP-PE & $6(3-10)$ & $0(0-1)^{* * *}$ \\
NTISS & $5(1-10)$ & $0(0-1)^{* * *}$ \\
\hline
\end{tabular}

Where applicable, values are mean (SD). Non-normally distributed continuous variables are expressed as median (25th-75th centiles). Categorical variables are expressed as case numbers with percentages in parentheses.

Significant pairwise differences, Bonferroni corrected significance levels: ${ }^{*} 0.05<\mathrm{p}<0.01 ;{ }^{* *} 0.01<\mathrm{p}<0.0001 ;{ }^{* * *} \mathrm{p}<0.0001$.

NICU, Neonatal intensive care unit; SGA, small for gestational age; SNAPE-PE, score for neonatal acute physiology-perinatal extension; NTISS, neonatal therapeutic intervention scoring system.

Infants were classified on the basis of the results of the placental histology (HCA positive $v$ HCA negative). Placental histological examination was performed as previously described, ${ }^{17}$ and a histological diagnosis of chorioamnionitis was made in the presence of at least 10 polymorphonuclear leucocytes per field in 10 non-adjacent 400 power fields in membranes and/or placental chorionic plate. Pathologists were unaware of the results of neonatal clinical evaluations (interobserver agreement in identifying HCA: $\kappa$ value $=$ $0.96, \mathrm{SE}=0.013,95 \%$ confidence interval (CI) 0.94 to 0.98 ).

In pulse oximetry, a constant amount of light is absorbed by skin, other tissues, and non-pulsatile blood, whereas a variable amount of light is absorbed by pulsating arterial inflow. PI is a scaled numerical value derived from the magnitude of the pulsations displayed on the plethysmographic waveform, and is calculated as the percentage of pulsatile signal to non-pulsatile signal. PI values were assessed using a Masimo SET Radical pulse oximeter (Masimo Corp, Irvine, California, USA) ${ }^{13}$ with the sensor placed randomly on either of the feet. After the pulse wave was confirmed to be artefact-free, PI values were recorded for at least five minutes after delivery. Oxygen saturation $\left(\mathrm{Spo}_{2}\right)$, pulse rate, body (skin and core) temperature, and blood pressure were also measured. All the infants were examined under a radiant warmer, and Apgar scores at one and five minutes were routinely assigned by the newborn caregiver. Core (rectal) and peripheral (skin) temperature were measured using a Datascope Passport YSI 700 series temperature set (Datascope Co, Mahwah, New Jersey, USA). The same procedure was applied to the study of the case series representing the validation cohort (study phase 2). The caring physicians were immediately advised of PI values below the cut-off criterion during the first 10 minutes of life.

The study was approved by the local institutional review board, and informed consent was obtained from the parents of all the enrolled infants before the study.

\section{Data analysis}

Data were expressed as means (SD) for continuous normally distributed data and medians with interquartile range (25th and 75th centiles) for non-normal distributions. The $t$ test or MannWhitney $U$ test, and $\chi^{2}$ test or Fisher's exact test were used to compare continuous normally distributed data, nonparametric continuous data, and categorical data respectively. The predictive accuracy in identifying newborns with HCA for different PI cut-off values and one minute Apgar scores was calculated using receiver operating characteristic curve analysis. Predictive values were calculated on the basis of the observed frequencies of subclinical HCA. The MedCalc version 7.4.2 statistical software package (MedCalc Software, Mariakerke, Belgium) was used. A two sided p value of $<0.05$ was considered to be significant, and the Bonferroni corrected significance levels were used for multiple $t$ tests.

\section{RESULTS}

Significantly lower PI values at one minute $(\mathrm{p}<0.0001)$ and five minutes $(p<0.0001)$ after delivery were observed in the HCA group, together with a significantly lower one minute Apgar score $(\mathrm{p}=0.017)$ (table 1$)$. The infants with HCA had significantly lower cord blood base excess $(p=0.0001)$, higher neonatal intensive care unit admission rate $(\mathrm{p}=$ $0.0001)$, endotracheal intubation in the delivery room ( $\mathrm{p}=$ $0.017)$, SNAP-PE $(p<0.0001)$ and NTISS $(p<0.0001)$ scores than the HCA negative newborns. On the other hand, cord blood $\mathrm{pH}$, prevalence of small for gestational age, five minute Apgar score, one minute and five minute $\mathrm{Spo}_{2}$, one minute and five minute pulse rate, one minute and five minute skin temperature, and one minute and five minute core temperature were not significantly different between the groups $(p \geqslant 0.26)$. At this stage of the study, one minute PI $\leqslant 1.74$ and five minute PI $\leqslant 2.18$ showed $100 \%$ sensitivity and

Table 2 Results of screening for subclinical histological chorioamnionitis (HCA) by applying the one minute and five minute perfusion index (PI) cut-off values generated by study phase 1 to an unselected term newborn population recruited prospectively

\begin{tabular}{|c|c|c|}
\hline \multirow[b]{2}{*}{ Variable } & \multicolumn{2}{|c|}{$1 \mathrm{~min}$ and $5 \mathrm{~min} \mathrm{PI}<$ cut-off criteria } \\
\hline & Yes & No \\
\hline $\begin{array}{l}\text { Number of cases } \\
\text { M:F } \\
\text { Gestational age (weeks) } \\
\text { Birth weight (g) } \\
1 \text { min perfusion index } \\
5 \text { min perfusion index } \\
\text { Confirmative diagnosis of HCA } \\
1 \text { min Apgar score } \\
5 \text { min Apgar score } \\
1 \text { min core temperature }\left({ }^{\circ} \mathrm{C}\right) \\
1 \text { min skin temperature }\left({ }^{\circ} \mathrm{C}\right) \\
5 \text { min core temperature }\left({ }^{\circ} \mathrm{C}\right) \\
5 \text { min skin temperature }\left({ }^{\circ} \mathrm{C}\right) \\
\text { Cord blood pH } \\
\text { Cord blood base excess } \\
\text { Admission to NICU } \\
\text { SGA (birth weight }<3 \text { rd centile) } \\
\text { Endotracheal intubation } \\
\text { SNAP-PE } \\
\text { NTISS }\end{array}$ & $\begin{array}{l}32 \\
19: 13 \\
39.3(1.5) \\
3225(375) \\
1.53(0.98) \\
1.98(1.0) \\
30(93.7 \%) \\
5(4-9) \\
9(7-9) \\
37.0(0.20) \\
34.4(0.34) \\
37.0(0.19) \\
34.8(0.31) \\
7.303(0.185) \\
-6.1(4.4) \\
2(6.2 \%) \\
2(6.2 \%) \\
1(3.3 \%) \\
3(3-8) \\
2(1-5)\end{array}$ & $\begin{array}{l}297 \\
149: 148 \\
39.6(1.0) \\
3380(440) \\
3.95(1.2)^{* * *} \\
4.02(2.14)^{\star * *} \\
0 / 297(0 \%) \\
9(8-10)^{*} \\
9(9-10) \\
37.1(0.15) \\
34.7(0.25) \\
37.03(0.31) \\
34.8(0.58) \\
7.335(0.12) \\
-1.2(3.8)^{* * *} \\
14(4.71 \%) \\
11(3.34 \%) \\
1(0.9 \%) \\
0(0-1)^{* * *} \\
0(0-1)^{* * *}\end{array}$ \\
\hline \multicolumn{3}{|c|}{$\begin{array}{l}\text { Where applicable, values are mean (SD). Non-normally distributed } \\
\text { continuous variables are expressed as median ( } 25 \text { th-75th centiles). } \\
\text { Categorical variables are expressed as case numbers with percentages in } \\
\text { parentheses. } \\
\text { Significant pairwise differences, Bonferroni corrected significance levels: } \\
{ }^{*} 0.05<p<0.01 ;{ }^{* *} 0.01<p<0.0001 \text {; }{ }^{* *} p<0.0001 \text {. } \\
\text { NICU, Neonatal intensive care unit; SGA, small for gestational age; } \\
\text { SNAPE-PE, score for neonatal acute physiology-perinatal extension; } \\
\text { NTISS, neonatal therapeutic intervention scoring system. }\end{array}$} \\
\hline
\end{tabular}


specificity in discriminating HCA positive from HCA negative cases. In the prospective validation study, applying the generated PI cut-off values (one minute PI $\leqslant 1.74$, five minute PI $\leqslant 2.18$ ) to the validation cohort resulted in a total of 30 $(9.12 \%)$ true positive, two $(0.61 \%)$ false positive, $0(0 \%)$ false negative, and $297(90.27 \%)$ true negative results. The accuracy of the one minute and five minute PI screenings showed $100 \%$ sensitivity (95\% CI $88.3 \%$ to $100 \%$ ), $99.4 \%$ specificity (95\% CI 97.8 to 99.9 ), $93.7 \%$ positive predictive, and $100 \%$ negative predictive value in identifying HCA. The two infants showing false positive results were subsequently diagnosed as having a congenital heart defect (Ebstein anomaly (median PI value in the first five minutes 0.68 (interquartile range $0.53-0.83)$ ) and non-ductal dependent aortic coarctation (median PI value in the first five minutes 0.75 (interquartile range $0.54-0.96)$ ). No specific differences between the PI evaluations at one and five minutes were observed. The application of PI screening to study phase 2 resulted in a significantly lower rate of admission to the neonatal intensive care unit of the newborns with HCA ( $\mathrm{p}=$ 0.01204 , relative risk $=0.21,95 \% \mathrm{CI}=0.052$ to 0.87 ), and significantly lower initial illness severity $(\mathrm{p}<0.0001)$ and therapeutic intensity $(p=0.0006)$ than those observed in the HCA cohort from study phase 1 (table 2). In contrast, sex, gestational age, proportion of small for gestational age birth, birth weight, one minute and five minute Apgar scores, cord blood base excess, endotracheal intubation rate, one minute and five minute $\mathrm{SpO}_{2}$, one minute and five minute pulse rate, one minute and five minute skin temperature, and one minute and five minute core temperature were comparable between the two HCA cohorts $(p \geqslant 0.39)$.

\section{DISCUSSION}

Our findings indicate that HCA positive term infants with subclinical disease show lower PI values from the first minutes of life than control neonates, and have worse short term outcomes. In addition, earlier HCA detection was associated with a reduction in neonatal illness severity. These findings, as well as confirming the importance of PI monitoring in the high risk newborn, ${ }^{13}$ provide, for the first time, an easily applicable and non-invasive diagnostic tool for the early screening of perinatal inflammatory disease, suggesting its use in newborn monitoring right from the delivery room. As a consequence, early PI monitoring could help in the early detection of these high risk newborns. The data from the validation arm of this study suggest that early HCA screening leads to a significant reduction in initial illness severity and therapeutic intensity at 24 hours. The decreased rate of admission to the neonatal intensive care unit observed during study phase 2 may be explained by a higher degree of monitoring in these high risk newborns generated by the positivity of an early marker of perinatal disease, associated with earlier, less invasive management of respiratory distress syndrome. The decreased initial illness severity and therapeutic intensity may be explained by similar reasoning. On the other hand, it is conceivable that the lack of changes in the Apgar scores, cord blood $\mathrm{pH}$, and base deficit between the HCA positive infants of the two study phases may be due to their dependence on prenatal rather than postnatal factors. The reasons for the significantly lower PI readings observed in the infants with HCA remain to be elucidated. However, HCA is associated with a fetal systemic inflammatory response, ${ }^{18}{ }^{19}$ similar to that observed in sepsis. ${ }^{2021}$ Systemic vasoconstriction has been reported in neonatal experimental models of sepsis, ${ }^{20}$ and an increased post-occlusive hyperaemia in septic neonates, compatible with systemic vasoconstriction, has been described. ${ }^{21}$ Therefore it is conceivable that local skin vasoconstriction and/or peripheral microcirculatory changes

\section{What is already known on this topic}

- Chorioamnionitis (CA) is known to be an often subclinical, major cause of premature labour and delivery leading to premature birth with increased neonatal morbidity and mortality

- In contrast, very little is known about the effects of CA at term, and no early marker for a subclinical form at term is available

\section{What this study adds}

- This study indicates for the first time that a measurable change in peripheral perfusion occurs in the early postnatal adaptation in term newborns with subclinical CA

- Early monitoring after birth of the pulse oximeter perfusion index accurately identifies affected newborns and positively influences short term neonatal outcome

may explain the observed findings. The recent evidence of early postnatal changes in skin colour in term newborns with subclinical HCA seems to support this hypothesis. ${ }^{22}$ Although the value of this measure in influencing longer term outcome has not yet been demonstrated, our study suggests that a low PI value allows early and accurate identification of terms infants with HCA.

\section{ACKNOWLEDGEMENTS}

We thank Ombretta Bugiani and Roberto Faleri (Central Medical Library, University of Siena) for online research assistance, and Ruth McBride for expert editorial assistance.

\section{Authors' affiliations}

C De Felice, Neonatal Intensive Care Unit, Azienda Ospedaliera Universitaria Senese, Siena, Italy

S Parrini, Department of Odontostomatology, Azienda Ospedaliera Universitaria Senese

A Del Vecchio, G Latini, Division of Neonatology, Perrino Hospital, Brindisi, Italy

M Criscuolo, A Lozupone, Pathology Unit, Perrino Hospital G Latini, Clinical Physiology Institute, National Research Council of Italy (IFC-CNR), Lecce Section, Italy

Competing interests: none declared

\section{REFERENCES}

1 De Felice C, Toti P, Laurini RN, et al. Early neonatal brain damage in histologic chorioamnionitis. J Pediatr 2001;138:101-4.

2 Hagberg H, Wennerholm UB, Savman K. Sequelae of chorioamnionitis. Curr Opin Infect Dis 2002;15:301-6.

3 Wu YW, Escobar GJ, Grether JK, et al. Chorioamnionitis and cerebral palsy in term and near-term infants. JAMA 2003;290:2677-84.

4 Willoughby RE Jr, Nelson KB. Chorioamnionitis and brain injury. Clin Perinatol 2002;29:603-21

5 Shalak LF, Laptook AR, Jafri HS, et al. Clinical chorioamnionitis, elevated cytokines, and brain injury in term infants. Pediatrics 2002;110:673-80.

6 Presta G, Rosati E, Giannuzzi R, et al. Prolonged fetal bradycardia as the presenting clinical sign in Streptococcus agalactiae chorioamnionitis. J Perinat Med 2004;32:535-7

7 Alexander JM, Mclntire DM, Leveno KJ. Chorioamnionitis and the prognosis for term infants. Obstet Gynecol 1999;94:274-8.

8 Negishi H, Matsuda T, Okuyama K, et al. Staphylococcus aureus causing chorioamnionitis and fetal death with intact membranes at term. A case report. J Reprod Med 1998;43:397-400.

9 Williams MC, O'Brien WF, Nelson RN, et al. Histologic chorioamnionitis is associated with fetal growth restriction in term and preterm infants. Am J Obstet Gynecol 2000;183:1094-9. 
10 Garland SM, Ni Chuileannain F, Satzke C, et al. Mechanisms, organisms and markers of infection in pregnancy. J Reprod Immunol 2002;57:169-83.

11 Toti P, De Felice C. Chorioamnionitis and fetal/neonatal brain injury. Biol Neonate 2001:79:201-4.

12 Aoyagi T, Miyasaka K. Pulse oximetry: its invention, contribution to medicine, and future tasks. Anesth Analg 2002;94(suppl 1):S1-3.

13 De Felice C, Latini G, Vacca P, et al. The pulse oximeter perfusion index as a predictor for high illness severity in neonates. Eur J Pediatr 2002;161:561-2.

14 Lima AP, Beelen P, Bakker J. Use of a peripheral perfusion index derived from the pulse oximetry signal as a noninvasive indicator of perfusion. Crit Care Med 2002;30:1210-13.

15 Gray JE, Richardson DK, McCormick MC, et al. Neonatal therapeutic intervention scoring system: a therapy-based severity-of-illness index. Pediatrics 1992;90:561-7.

16 Richardson DK, Phibbs CS, Gray JE, et al. Birth weight and illness severity: independent predictors of neonatal mortality. Pediatrics 1993;91:969-75.
17 Toti P, De Felice C, Stumpo M, et al. Acute thymic involution in fetuses and neonates with chorioamnionitis. Hum Pathol 2000;31:1121-8.

18 Gomez R, Romero R, Ghezzi $F$, et al. The fetal inflammatory response syndrome. Am J Obstet Gynecol 1998;179:194-202.

19 Dammann O, Kuban KC, Leviton A. Perinatal infection, fetal inflammatory response, white matter damage, and cognitive limitations in children born preterm. Ment Retard Dev Disabil Res Rev 2002;8:46-50.

20 Del Moral T, Goldberg RN, Suguihara C, et al. Effects of pentoxifylline on the cardiovascular manifestations of group $B$ streptococcal sepsis in the piglet. Pediatr Res 1992;31:596-600.

21 Poschl JM, Weiss T, Fallahi F, et al. Reactive hyperemia of skin microcirculation in septic neonates. Acta Paediatr 1994:83:808-11.

22 De Felice C, Vacca P, Del Vecchio A, et al. Early postnatal skin colour changes in term newborns with subclinical histological chorioamnionitis. Eur J Pediatr $2004 ; 163: 550-4$ 